\title{
Guest Editorial: Web-based services and information systems
}

\author{
Lei Chen • Zhiguo Gong • Qing Li
}

Received: 29 May 2012 / Revised: 29 May 2012

Accepted: 6 June 2012 / Published online: 27 June 2012

(C) Springer Science+Business Media, LLC 2012

The rapid growth of the Web data requires new technologies for design, implementation, and management of Web-based services and information systems. It is clear that traditional relational database techniques cannot meet the requirement of such large scale, distributed, heterogeneous data in the Web. Therefore, it is urgent and essential to provide effective and efficient techniques for modeling, specifying, retrieving, integrating, exchanging, querying, archiving, managing, analyzing, cleaning and securing the data on the Web. This special issue contains eight papers addressing some of the above-mentioned issues, as briefly discussed below.

In "Top-k Answers for XML Keyword Queries", the authors present an approach for identifying the relevant answers of a query by combining the mutual information concept and skyline semantics. Ranking criteria are proposed to select top-k results. Algorithms are introduced to efficiently identify the top-k dominant results. The proposed approach outperforms exisiting approaches with respect to a variety of evaluation metrics.

The paper, entitled "Automatic extraction of OWL ontologies from UML class diagrams: a semantics-preserving approach", investigates a formal approach to automatic, semanticspreserving translation from a UML 2 class diagram to an OWL DL ontology. The proposed translation algorithm and UML2OWL tool capture richer knowledge of UML constructs and constraints compared with existing solutions. Contributions also include the formal proof for the semantics preservation of the translation, and the theoretical analysis and experimental validation of the time complexity of the algorithm.

\footnotetext{
L. Chen $(\bowtie)$

Department of Computer Science and Engineering, Hong Kong University of Science and Technology,

Clear Water Bay, Kowloon, Hong Kong, China

e-mail: leichen@cse.ust.hk

Z. Gong

Department of Computer and Information Sciences, University of Macau, Macau, China e-mail: fstzgg@umac.mo

Q. Li

Department of Computer Science, City University of Hong Kong, Kowloon, Hong Kong, China e-mail: itqli@cityu.edu.hk
} 
In trust management systems, approaches are needed to use a small set of data to represent a large volume of trust ratings for a service provider with a long service period and well preserve trust features. In "The study of trust vector based trust rating aggregation in service-oriented environments", the authors propose one greedy and two optimal multiple time interval (MTI) analysis algorithms. The greedy algorithm is efficient, while the optimal algorithms can yield the minimal set of time intervals, within each of which a trust vector can be obtained and can well represent all the corresponding ratings.

Collaborative tagging becomes a common feature of current web sites, facilitating ordinary users to annotate and represent online resources. As a hierarchical concept structure to represent subsumption relationships, automatically extracted taxonomies become a viable method to manage collaborative and dynamic tags. In "Evolutionary taxonomy construction from dynamic tag space", the problem of evolutionary taxonomy generation over a large collection of tags is addressed. The authors develop a context-aware edge selection algorithm for taxonomy extraction, and propose several strategies for evolutionary taxonomy fusion, which smooths the newly generated taxonomy with prior ones. The empirical results over a large real-life tagging dataset (i.e., Del.ici.ous) verify the effectiveness and efficiency of their proposed approach.

The paper, titled "Exploring latent browsing graph for question answering recommendation", gives a new framework for Question Answering systems. In the proposed framework, user click streams and browsing behaviors among QA pages are caught and modeled as a QA Latent Browsing Graph, based on which, two scores, the importance score of QA pages and the relevance score of QA pages are computed. The QA pages are then ranked with their scores.

In "Path-oriented Keyword Search over Graph-modeled Web Data," the authors present a novel approach for keyword search where query answers over graph-modeled web data are constructed in a ranked order by combining the best results in the first set of generated results. The paper provides both experimental and theoretical evidence that the proposed approach is superior to state of the art systems in terms of efficiency and quality of results.

The paper, titled "A novel QoS model and computation framework in web service selection", presents a novel QoS (Quality of Service) model to solve the problem of web service selection with QoS constraints. The key idea of the model is to relax users' QoS constraints and try to find the most possible services satisfying users' QoS requirements. Based on the proposed QoS framework, various algorithms are proposed and provided for making service selection on individual and composite services.

Use of the guidelines, algorithms and detailed software engineering techniques presented, would open up new application areas such as industrial control systems, avionics, robotics and financial trading systems to the use of web services as a middleware platform. The last paper, titled "Building Web Services Middleware with Predictable Service Execution", proposes an approach to introduce predictability into web service execution in stand-alone and cluster-based web service middleware. Service invocations are explicitly selected and scheduled to meet user requested processing deadlines. In clusters, dispatching decisions are made to maximize deadline achievement rates. The authors have put forward a through analysis of the practical aspects of achieving predictability in web services middleware, along with discussions of the challenges and possible solutions.

Finally, we would like to thank those individuals who have helped make this special issue possible. Most important of all, we would like to thank the authors who submitted manuscripts for consideration, and the many dedicated, anonymous reviewers who helped us arrive at our final decision. 